\title{
A CLASS OF EXCHANGE RINGS
}

\author{
TSIU-KWEN LEE \\ Department of Mathematics, National Taiwan University, Taipei 106, Taiwan. Member of Mathematics \\ Division (Taipei Office), National Center for Theoretical Sciences \\ e-mail:tklee@math.ntu.edu.tw \\ and YIQIANG ZHOU* \\ Department of Mathematics and Statistics, Memorial University of Newfoundland, St. John's, \\ Newfoundland A1C 5S7, Canada \\ e-mail: zhou@math.mun.ca
}

(Received 26 August 2007; accepted 31 March 2008)

\begin{abstract}
It is well known that a ring $R$ is an exchange ring iff, for any $a \in R$, $a-e \in\left(a^{2}-a\right) R$ for some $e^{2}=e \in R$ iff, for any $a \in R, a-e \in R\left(a^{2}-a\right)$ for some $e^{2}=e \in R$. The paper is devoted to a study of the rings $R$ satisfying the condition that for each $a \in R, a-e \in\left(a^{2}-a\right) R$ for a unique $e^{2}=e \in R$. This condition is not leftright symmetric. The uniquely clean rings discussed in (W. K. Nicholson and Y. Zhou, Rings in which elements are uniquely the sum of an idempotent and a unit, Glasgow Math. J. 46 (2004), 227-236) satisfy this condition. These rings are characterized as the semi-boolean rings with a restricted commutativity for idempotents, where a ring $R$ is semi-boolean iff $R / J(R)$ is boolean and idempotents lift modulo $J(R)$ (or equivalently, $R$ is an exchange ring for which any non-zero idempotent is not the sum of two units). Various basic properties of these rings are developed, and a number of illustrative examples are given.
\end{abstract}

1991 Mathematics Subject Classification. Primary 16U99.

1. Introduction. Rings $R$ are associative with unity. We write $J(R)$ and $U(R)$ for the Jacobson radical and the group of units of $R$. In [13], Nicholson observed an interesting relation between elements in a ring $R$,

$$
a-e \in U(R) \text { for some } e^{2}=e \Longrightarrow a-f \in\left(a^{2}-a\right) R \text { for some } f^{2}=f .
$$

Consequently, every clean ring is an exchange ring, where a ring is called clean if each of its elements is the sum of a unit and an idempotent. Exchange rings have been extensively studied by many authors (for example, see $[2-5,7,9,10,12-14,19,20,22$, 23]). Clean rings is an active subject recently. A ring $R$ is called uniquely clean if for each $a \in R, a-e \in U(R)$ for a unique idempotent $e$. As discussed in $[\mathbf{1}, \mathbf{6}, \mathbf{1 6}, \mathbf{1 8}$, the uniquely clean rings turned out to be a natural generalization of boolean rings and their structure is well understood: a ring $R$ is uniquely clean iff $R / J(R)$ is boolean, idempotents lift modulo $J(R)$ and the idempotents of $R$ are central.

In this paper, we are motivated to study the rings $R$ satisfying the condition that for each $a \in R, a-f \in\left(a^{2}-a\right) R$ for a unique idempotent $f$. We prove that this condition

*Corresponding author. 
is not left-right symmetric (see Example 28). Every uniquely clean ring satisfies this condition. The rings with this condition are characterized as the semi-boolean rings with a restricted commutativity for idempotents (see Theorem 17), where a ring $R$ is semi-boolean iff $R / J(R)$ is boolean and idempotents lift modulo $J(R)$ [or equivalently, $R$ is an exchange ring for which any non-zero idempotent is not the sum of two units (see Theorem 13)]. Various basic properties of these rings are developed, and a number of illustrative examples are given.

The ring of integers modulo $n$ is denoted by $\mathbb{Z}_{n}$. We write $\mathbb{M}_{n}(R)$ [resp., $\mathbb{T}_{n}(R)$ ] for the ring of all $n \times n$ matrices (resp., all upper triangular $n \times n$ matrices) over $R$. The ring of polynomials (resp., power series) in indeterminate $x$ over a ring $R$ is denoted by $R[x]$ [resp., $R[[x]]]$. For an endomorphism $\sigma$ of a ring $R, R[x ; \sigma]$ and $R[[x ; \sigma]]$ denote the (left) skew polynomial ring and (left) skew power series ring, in which the multiplication is subjected to the condition that $x r=\sigma(r) x$ for all $r \in R$.

2. A few easy lemmas. The first two lemmas are implicit in [13].

LemMA 1. Let $R$ be a ring and $x, a \in R$. Then

$$
a \in x R, 1-a \in(1-x) R \Leftrightarrow x-a \in\left(x^{2}-x\right) R .
$$

Proof. " $\Rightarrow " . x-a=x-x a+x a-a=x(1-a)+(x-1) a \in\left(x^{2}-x\right) R$.

" $\Leftarrow "$. If $x-a \in\left(x^{2}-x\right) R$, then $a \in x R$ and $1-a=(1-x)+(x-a)$ $\in(1-x) R$.

LemMA 2. Let $a, e^{2}=e, u, v \in R$. The following hold:

(1) If $a=e+u$ and $u v=1$, then $a-u(1-e) v=\left(a^{2}-a\right) v$.

(2) If $a=e+u$ and $v u=1$, then $a-v(1-e) u=v\left(a^{2}-a\right)$.

(3) If $a=e+u$ where $u \in U(R)$, then $a-u(1-e) u^{-1}=\left(a^{2}-a\right) u^{-1}$ and $a-u^{-1}(1-e) u=u^{-1}\left(a^{2}-a\right)$.

Proof. The verification of (1) and (2) is straightforward, and (3) follows by (1) and (2).

Lemma 3. Let $a, e^{2}=e \in R$ with ea $=$ ae. Then the following hold:

(1) $(a-e) R=R \Leftrightarrow a-(1-e) \in\left(a^{2}-a\right) R$.

(2) $R(a-e)=R \Leftrightarrow a-(1-e) \in R\left(a^{2}-a\right)$.

Proof. (1) ' $\Rightarrow$ '. Let $u=a-e$ and $u v=1$ with $v \in R$. Then $a-(1-e)=a-$ $(1-e) u v=a-u(1-e) v=\left(a^{2}-a\right) v$ by Lemma $2(1)$.

' $\Leftarrow$ '. Suppose that $a-(1-e) \in\left(a^{2}-a\right) R$. Then $1-e \in a R$ and $e \in(1-a) R$ by Lemma 1. Write $1-e=a r$ and $e=(1-a) s$ where $r, s \in R$. Then $1-e=(1-e) \operatorname{ar}(1-$ $e)=a(1-e) r(1-e)$ and $e=e(1-a) s e=(1-a) e s e$. So we may assume that $r=(1-$ $e) r=r(1-e)$ and $s=e s=s e$. Then $(a-e)(r-s)=a r-a s-e r+e s=1-e-a s+$ $s=1-e+(1-a) s=1-e+e=1$. So $(a-e) R=R$.

(2) The proof is similar to (1).

COROLlaRY 4. The equivalences (1) $\Leftrightarrow$ (2) and (3) $\Leftrightarrow$ (4) hold for a ring $R$.

(1) For any $a \in R$, there exist $e^{2}=e \in R$ and $u \in R$ with $u R=R$ such that $a=e+u$ and $e a=a e$.

(2) For any $a \in R$, there exists $e^{2}=e \in R$ such that $a-e \in\left(a^{2}-a\right) R$ and $e a=a e$. 
(3) For any $a \in R$, there exist $e^{2}=e \in R$ and $u \in R$ with $R u=R$ such that $a=e+u$ and $e a=a e$.

(4) For any $a \in R$, there exists $e^{2}=e \in R$ such that $a-e \in R\left(a^{2}-a\right)$ and $e a=a e$.

An element $a \in R$ is called strongly clean if $a=e+u$ and $e a=a e$ where $e^{2}=e$ and $u \in U(R)$, and a ring is called strongly clean if each of its elements is strongly clean [15].

COROLlARY 5. The following are equivalent for $a \in R$ :

(1) a is strongly clean in $R$.

(2) There exists $e^{2}=e \in R$ such that $e \in a R \cap R a, 1-e \in(1-a) R \cap R(1-a)$ and $e a=a e$.

(3) There exists $e^{2}=e \in R$ such that $a-e \in\left(a^{2}-a\right) R \cap R\left(a^{2}-a\right)$ and ae $=e a$.

Proof. (1) $\Rightarrow$ (3). Suppose that $a$ is strongly clean in $R$ and write $a=e+u$ where $e^{2}=e, u \in U(R)$ and $e a=a e$. By Lemma 3, $a-(1-e) \in\left(a^{2}-a\right) R \cap R\left(a^{2}-a\right)$. So (3) holds. clean.

(3) $\Rightarrow(1)$. By Lemma 3, (3) implies that $a-(1-e)$ is a unit of $R$, so $a$ is strongly

(2) $\Leftrightarrow(3)$. This is by Lemma 1 and by its left version.

Every strongly clean element $a \in R$ satisfies both (1) and (2) of Lemma 3. But neither of (1) and (2) of Lemma 3 implies that $a$ is strongly clean, as shown in the next example [21].

EXAMPLE 6. [21]. Let $V$ be a right vector space of countably infinite dimension over a division ring $D$ with $\left\{v_{i}: i=1,2, \ldots\right\}$ a basis. Define $\sigma: V \rightarrow V$ by $\sigma\left(v_{i}\right)=v_{i+1}$ for each $i \geq 1$. Let $R=\operatorname{End}\left(V_{D}\right)$ be the endomorphism ring and $S=R^{o}$ be the opposite ring of $R$. Then the following hold:

(1) $\sigma$ is left invertible in $R$, but $\sigma$ is not strongly clean in $R$.

(2) $\sigma$ is right invertible in $S$, but $\sigma$ is not strongly clean in $S$.

Proof. (1) Define $\rho: V \rightarrow V$ by $\rho\left(v_{i+1}\right)=v_{i}$ for $i \geq 1$ and $\rho\left(v_{1}\right)=0$. Clearly, $\rho \circ \sigma=1_{V}$, so $\sigma$ is left invertible in $\operatorname{End}\left(V_{D}\right)$. Suppose that $\sigma=e+u$ and $e \circ u=u \circ e$ where $e^{2}=e \in \operatorname{End}\left(V_{D}\right)$ and $u$ is a unit of $\operatorname{End}\left(V_{D}\right)$. Then $1-e=\sigma \circ(1-e) \circ u^{-1}$. So

$$
v_{i}-e\left(v_{i}\right) \in \sigma(V)=\operatorname{span}\left(v_{2}, v_{3}, \ldots\right) \text { for each } i \geq 1 .
$$

Since $u$ is a unit of $R$, there exists $v=v_{1} a_{1}+\cdots+v_{n} a_{n} \in V$ such that

$$
\begin{aligned}
v_{1} & =u(v)=(\sigma-e)(v) \\
& =v_{2} a_{1}+v_{3} a_{2}+\cdots+v_{n+1} a_{n}-e\left(v_{1}\right) a_{1}-e\left(v_{2}\right) a_{2}-\cdots-e\left(v_{n}\right) a_{n} .
\end{aligned}
$$

Because of (2.1), it follows that $a_{1}=-1$. Applying $e$ to both sides of (2.2) yields that

$$
\begin{aligned}
0 & =e\left(v_{2}\right)\left(a_{1}-a_{2}\right)+e\left(v_{3}\right)\left(a_{2}-a_{3}\right)+\cdots+e\left(v_{n}\right)\left(a_{n-1}-a_{n}\right)+e\left(v_{n+1}\right) a_{n} \\
& =\sigma\left(e\left(v_{1}\right)\left(a_{1}-a_{2}\right)+e\left(v_{2}\right)\left(a_{2}-a_{3}\right)+\cdots+e\left(v_{n-1}\right)\left(a_{n-1}-a_{n}\right)+e\left(v_{n}\right) a_{n}\right) .
\end{aligned}
$$

Since $\sigma$ is one-to-one, it follows that

$$
0=e\left(v_{1}\right)\left(a_{1}-a_{2}\right)+e\left(v_{2}\right)\left(a_{2}-a_{3}\right)+\cdots+e\left(v_{n-1}\right)\left(a_{n-1}-a_{n}\right)+e\left(v_{n}\right) a_{n} .
$$


Again by (2.1), one obtains $a_{1}-a_{2}=0$ and so

$$
\begin{aligned}
0 & =e\left(v_{2}\right)\left(a_{2}-a_{3}\right)+\cdots+e\left(v_{n-1}\right)\left(a_{n-1}-a_{n}\right)+e\left(v_{n}\right) a_{n} \\
& =\sigma\left(e\left(v_{1}\right)\left(a_{2}-a_{3}\right)+\cdots+e\left(v_{n-2}\right)\left(a_{n-1}-a_{n}\right)+e\left(v_{n-1}\right) a_{n}\right)
\end{aligned}
$$

Repeating the process above, one obtains $a_{2}-a_{3}=0$. A simple induction clearly shows that $a_{1}=-1, a_{i}-a_{i+1}=0$ for $i=1, \ldots, n-1$ and $a_{n}=0$. This is a contradiction.

(2) follows by (1).

QUESTION 1. Suppose that for any $a \in R$, there exists $e^{2}=e \in R$ such that $e a=a e$ and $a-e \in\left(a^{2}-a\right) R$ (resp., $\left.a-e \in R\left(a^{2}-a\right)\right)$. Is $R$ strongly clean?

3. Right uniquely exchange rings. An element $a$ in a ring $R$ is called a right uniquely exchange element if $\exists \mid e^{2}=e \in R$ such that $e \in a R$ and $1-e \in(1-a) R$ (or equivalently, $\exists \mid e^{2}=e \in R$ such that $\left.a-e \in\left(a^{2}-a\right) R\right)$. The ring $R$ is called a right uniquely exchange ring if every element of $R$ is a right uniquely exchange element. Left uniquely exchange rings (resp., elements) are defined analogously.

In the proof of [14, Proposition], Nicholson actually showed that, for $a \in R$, $\exists e^{2}=e \in R$ such that $a-e \in\left(a^{2}-a\right) R$ iff $\exists f^{2}=f \in R$ such that $a-f \in R\left(a^{2}-a\right)$. But a right uniquely exchange element need not be a left uniquely exchange element. Indeed, a right uniquely exchange ring need not be a left uniquely exchange ring (see Example 28).

EXAMPLE 7. Let $R$ be a ring and $a \in R$.

(1) Idempotents of $R$ and elements in $J(R)$ all are left and right uniquely exchange elements.

(2) $a$ is a right uniquely exchange element iff so is $1-a$.

Proof. (1) If $a^{2}=a \in R$, let $e=a$ and we have $e \in a R$ and $1-e \in(1-a) R$. If $f \in a R$ and $1-f \in(1-a) R$ with $f^{2}=f$, then $(1-a) f=0$ and $a(1-f)=0$. It follows that $f=a$.

If $a \in J(R)$, let $e=0$ and then $e \in a R$ and $1-e \in(1-a) R$. If $f \in a R$ and $1-f \in$ $(1-a) R$ with $f^{2}=f$, then $f \in J(R)$. Thus $f=0$.

(2) This follows from the fact that $a-e \in\left(a^{2}-a\right) R \Leftrightarrow(1-a)-(1-e) \in[(1-$ $\left.a)^{2}-(1-a)\right] R$.

A ring $R$ is called uniquely clean if every element of $R$ is uniquely the sum of a unit and an idempotent. These rings are the topic of $[\mathbf{1}, \mathbf{6}, \mathbf{1 6}, \mathbf{1 8}]$. They are characterized in [16] as the rings $R$ such that $R / J(R)$ is boolean, idempotents lift modulo $J(R)$, and all idempotents in $R$ are central.

EXAMPLE 8. Every uniquely clean ring is a right uniquely exchange ring.

Proof. For $a \in R$, there exists $e^{2}=e \in a R$ with $1-e \in(1-a) R$. Suppose that $f^{2}=f \in a R$ with $1-f \in(1-a) R$. Then $e-f \in a R \cap(1-a) R$. By [16, Theorem 20], $a R \cap(1-a) R \subseteq J(R)$. So $e-f \in J(R)$. From $e+0=f+(e-f)$, it follows by [16, Theorem 20(4)] that $e=f$. So $R$ is a right uniquely exchange ring.

However, a right uniquely exchange ring need not be uniquely clean. 
EXAMPLE 9. Let $R=\left(\begin{array}{cc}\mathbb{Z}_{2} & \mathbb{Z}_{2} \\ 0 & \mathbb{Z}_{2}\end{array}\right)$. Then $R$ is clearly an exchange ring, but $R$ is not uniquely clean because it has non-central idempotents. Note that $a=\left(\begin{array}{ll}\overline{1} & \overline{1} \\ 0 & \overline{1}\end{array}\right)$ is the only element which is neither an idempotent nor an element in $J(R)$. Since $1-a \in J(R)$, the unique idempotent $e \in a R$ with $1-e \in(1-a) R$ is $e=1$. So $R$ is a right uniquely exchange ring in view of Example 7.

Proposition 10. Let $R$ be a local ring. The following are equivalent:

(1) $R$ is a right uniquely exchange ring.

(2) $R$ is a uniquely clean ring.

(3) $R / J(R) \cong \mathbb{Z}_{2}$.

Proof. (3) $\Rightarrow(2) \Rightarrow(1)$. This is true by [16, Theorem 15].

$(1) \Rightarrow(3)$. Let $u \notin J(R)$. We show that $1-u \in J(R)$. If $1-u \notin J(R)$ then $1-u \in$ $U(R)$ and $1-(1-u)=u \in U(R)$. So for $a=1-u$ and for $e \in\{0,1\}$, we have $e \in a R$ and $1-e \in(1-a) R$. So $a$ is not a right uniquely exchange element in $R$. Therefore $1-u \in J(R)$ and hence $\bar{u}=\overline{1}$.

Proposition 11. Let $R$ be a right uniquely exchange ring and $e^{2}=e \in R$. The following hold:

(1) eRe is a right uniquely exchange ring.

(2) If $e \neq 0$, then $e \neq u+v$ for all $u, v \in U(e R e)$. In particular, any matrix ring of size greater than 1 is not a right uniquely exchange ring.

Proof. (1) By [13, Proposition 1.10], $e$ Re is an exchange ring. If $a \in e R e$ and $a-e_{i} \in\left(a^{2}-a\right) e R e$ where $e_{i}^{2}=e_{i}$ for $i=1,2$, then $a-e_{i} \in\left(a^{2}-a\right) R$. Thus $e_{1}=e_{2}$ because $R$ is a right uniquely exchange ring.

(2) If $e=u+v$ with $u, v \in U(e R e)$, then $u-e=-v$. So $\left(u^{2}-u\right) e R e=$ $u(-v) e R e=e R e$. Hence $u-0, u-e \in\left(u^{2}-u\right)(e R e)$. This shows that $e R e$ is not a right uniquely exchange ring, contradicting (1). To show the last statement, note that the identity matrix of a $2 \times 2$ matrix ring is clearly the sum of two invertible matrices. So any $2 \times 2$ matrix ring is not a right uniquely exchange ring. Thus, any matrix ring of size greater than 1 is not a right uniquely exchange ring by (1).

4. Structures and characterizations. A ring $R$ is called semipotent if each left ideal (resp., right ideal) not contained in $J(R)$ contains a non-zero idempotent, and $R$ is called potent if, in addition, idempotents lift modulo $J(R)$. By [17, Example 25], a semipotent ring need not be potent. Following [18], a ring $R$ is called semi-boolean if $R / J(R)$ is boolean and idempotents lift modulo $J(R)$.

A set $\left\{e_{i j}: 1 \leq i, j \leq n\right\}$ of non-zero elements of $R$ is said to be a system of $n^{2}$ matrix units if $e_{i j} e_{s t}=\delta_{j s} e_{i t}$, where $\delta_{j j}=1$ and $\delta_{j s}=0$ for $j \neq s$. In this case, $e:=\sum_{i=1}^{n} e_{i i}$ is an idempotent of $R$ and $e R e \cong \mathbb{M}_{n}(S)$ where $S=\left\{r \in e R e: r e_{i j}=e_{i j} r\right.$ for all $i, j=$ $1,2, \ldots, n\}$.

LEMMA 12. [11] Let $R$ be a semipotent ring with $J(R)=0$ and let $n>1$. If $a^{n}=0$ but $a^{n-1} \neq 0$ in $R$, then RaR contains a system of $n^{2}$-matrix units.

THEOREM 13. The following are equivalent for a ring $R$ :

(1) $R$ is a potent ring and, for any $0 \neq e^{2}=e \in R, e \neq u+v$ for all $u, v \in U(e R e)$.

(2) $R$ is a potent ring and every non-zero idempotent of $R$ is not the sum of two units. 
(3) $R$ is an exchange ring and every non-zero idempotent of $R$ is not the sum of two units.

(4) $R$ is a semi-boolean ring.

Proof. (4) $\Rightarrow(3) \Rightarrow(2)$. These are clear.

(2) $\Rightarrow(1)$. If $e^{2}=e=u+v$ where $u, v \in U(e R e)$, then $e=[u+(1-e)]+[v-$ $(1-e)]$ is the sum of two units of $R$.

$(1) \Rightarrow(4)$. Let $\bar{R}=R / J(R)$ and write $\bar{x}=x+J(R)$ for all $x \in R$.

Claim 1. $\bar{R}$ is a reduced ring.

Assume on the contrary that $\bar{a}^{2}=\overline{0}$ but $\bar{a} \neq \overline{0}$. Since $\bar{R}$ is semipotent, $\bar{R} \bar{a} \bar{R}$ contains a system of $2^{2}$-matrix units by Lemma 12. Thus, there exists $e \in R$ such that $\bar{e}^{2}=\bar{e} \in \bar{R}$ and $\bar{e} \bar{R} \bar{e} \cong \mathbb{M}_{2}(S)$ for some non-trivial ring $S$. Since idempotents lift modulo $J(R)$, we may assume that $e^{2}=e$. Clearly, the identity of $\mathbb{M}_{2}(S)$ can be written as the sum of two units of $\mathbb{M}_{2}(S)$, so $\bar{e}$ is the sum of two units of $\bar{e} \bar{R} \bar{e}$. Because $\bar{e} \bar{R} \bar{e} \cong e R e / J(e R e)$ and because units lift modulo the radical, it follows that $e$ is the sum of two units of $e R e$, a contradiction.

Claim 2. $a^{2}-a \in J(R)$ for each $a \in R$.

Suppose that $a^{2}-a \notin J(R)$ for some $a \in R$. Then there exists $0 \neq e=e^{2} \in\left(a^{2}-a\right) R$. Write $e=\left(a^{2}-a\right) b$ with $b e=b$, so $b\left(a^{2}-a\right)$ is an idempotent. By Claim 1, idempotents of $\bar{R}$ are central, so $\bar{b}\left(\bar{a}^{2}-\bar{a}\right)=\bar{b}\left(\bar{a}^{2}-\bar{a}\right) \bar{e}=\left(\bar{a}^{2}-\bar{a}\right)\left[\bar{b}\left(\bar{a}^{2}-\bar{a}\right)\right] \bar{b}=\bar{e}$. Thus, $\bar{b}$ and $\bar{e} \bar{a}$ are units of $\bar{e} \bar{R} \bar{e}$, and hence $\bar{e}=\bar{a}^{2} \bar{b}+(-\bar{a} \bar{b})$ is the sum of two units of $\bar{e} \bar{R} \bar{e}$. As in the proof of Claim 1, it follows that $e$ is the sum of two units of $e R e$, a contradiction.

There exist exchange rings $R$ such that $1 \neq u+v$ for any $u, v \in U(R)$, but they are not semi-boolean.

EXAMPLE 14. Let $R$ be the group ring $\mathbb{Z}_{2} C_{3}$ where $C_{3}$ is the cyclic group of order 3 . Then $R$ is a semi-simple ring by Maschke's Theorem, hence an exchange ring with $J(R)=0$. Assume that $1=u+v$ for some $u, v \in U(R)$. Applying the augmentation mapping to the equality gives that 1 is the sum of two units in $\mathbb{Z}_{2}$, a contradiction. However, $R$ is not boolean because it has non-trivial units.

The next example was suggested to us by the referee. It gives a ring $S$ with $S / J(S)$ boolean but such that idempotents do not lift modulo $J(S)$.

EXAMPLE 15. Let $k=\mathbb{Z}_{2}$ and let $k\langle x, y\rangle$ be the free algebra in the non-commuting variables $x$ and $y$. Let $R=k\langle x, y\rangle / y x=0$ be the factor ring of $k\langle x, y\rangle$ modulo the ideal generated by $y x$. Following [8], let $R_{\Sigma}$ be the universal localization of $R$ with respect to the set $\Sigma$ of all square matrices that become invertible under each of the following evaluations: $x \mapsto 0, y \mapsto 1$ and $x \mapsto 1, y \mapsto 0$. By [8, Corollary 6.4], $R_{\Sigma}$ has no nontrivial idempotents. However, $R_{\Sigma} / J\left(R_{\Sigma}\right) \cong k \times k$ by [8, Fact 4.1]. Thus, idempotents do not lift modulo $J\left(R_{\Sigma}\right)$, so $R_{\Sigma}$ is not semi-boolean.

A ring is called abelian if each of its idempotents is central.

Lemma 16. Let $R / J(R)$ be an abelian ring. Suppose that $a \in R$ and $e^{2}=e \in R$. If $a-e \in\left(a^{2}-a\right) R$ or $a-e \in R\left(a^{2}-a\right)$, then $a-(1-e)$ is a unit of $R$.

Proof. Let us assume that $a-e \in\left(a^{2}-a\right) R$. Then $e \in a R$ and $1-e \in(1-a) R$ by Lemma 1 . Write $e=a r$ with $r=r e$ and $1-e=(1-a) s$ with $s=s(1-e)$. Let $u=a-(1-e)$ and $v=r-s$. Because $R / J(R)$ is abelian, we have $\bar{e} \bar{r}=\bar{r}=\bar{r} \bar{e}$ and 
$\bar{e} \bar{s}=\bar{s} \bar{e}=0$ and so

$$
\begin{aligned}
\bar{u} \bar{v} & =[\bar{a}-(\overline{1}-\bar{e})](\bar{r}-\bar{s}) \\
& =\overline{a r}-(\overline{1}-\bar{e}) \bar{r}+(-\bar{a}+\overline{1}-\bar{e}) \bar{s} \\
& =\bar{e}+(\overline{1}-\bar{a}) \bar{s}=\bar{e}+(\overline{1}-\bar{e})=\overline{1} .
\end{aligned}
$$

So $\bar{v} \bar{u}=(\bar{v} \bar{u})(\bar{u} \bar{v})=\bar{u}(\bar{v} \bar{u}) \bar{v}=\overline{1}$. Hence $\bar{u}$ is a unit of $R / J(R)$, so $u$ is a unit of $R$.

A ring $R$ is said to satisfy $(*)$ if, whenever $a-e \in\left(a^{2}-a\right) R$ with $e^{2}=e$, we have $a e=e a$. It is interesting to compare part (4) of the next theorem with [16, Theorem 20(4)].

THEOREM 17. The following are equivalent for a ring $R$ :

1. $R$ is a right uniquely exchange ring.

2. $\forall a \in R, \exists \mid e^{2}=e$ such that $a-e \in U(R)$ and $a e=e a$, and $R$ satisfies $(*)$.

3. $\forall a \in R, \exists \mid e^{2}=e$ such that $a-e \in J(R)$ and $a e=e a$, and $R$ satisfies $(*)$.

4. $R$ is semi-boolean and the idempotents e satisfying $a-e \in\left(a^{2}-a\right) R$ commute with each other for each $a \in R$.

Proof. (1) $\Rightarrow$ (3). Suppose that (1) holds. Then, by Proposition 11, $R$ satisfies Theorem 13(1). So $R / J(R)$ is boolean by Theorem 13 .

We first show that $(*)$ holds. Suppose that $a-e \in\left(a^{2}-a\right) R$ where $e^{2}=e$. By Lemma $16, a=(1-e)+u$ where $u=a-(1-e) \in U(R)$. So $a-u e u^{-1} \in\left(a^{2}-a\right) R$ by Lemma 3. Thus, $e=u e u^{-1}$ or $e u=u e$ by (1). So $a e=e a$ and ( $*$ ) holds.

Claim. For $a, e^{2}=e \in R$,

$$
a-e \in J(R) \text { and } a e=e a \Leftrightarrow(a+1)-(1-e) \in\left[(a+1)^{2}-(a+1)\right] R .
$$

In fact, if $(a+1)-(1-e) \in\left[(a+1)^{2}-(a+1)\right] R$, then $(a+1)(1-e)=(1-e)(a+1)$ by $(*)$, i.e. $a e=e a$, and $u:=(a+1)-e \in U(R)$ by Lemma 16 . Thus, $\overline{1}=\bar{u}=\bar{a}+\overline{1}-$ $\bar{e}$, so $\bar{a}=\bar{e}$. Hence $a-e \in J(R)$ and $a e=e a$. Conversely, if $a-e \in J(R)$ and $a e=e a$, then $u:=(a+1)-e=1+(a-e) \in U(R)$. Thus $(a+1)-(1-e) \in\left[(a+1)^{2}-(a+\right.$ $1)] R$ by Lemma 3 . Now (3) follows.

(3) $\Rightarrow(2)$. Let $a \in R$. By (3), there exists $e^{2}=e \in R$ such that $(a-1)-e \in J(R)$ and $(a-1) e=e(a-1)$. Thus, $a-e=1+[(a-1)-e] \in U(R)$ with $a e=e a$. Suppose that $a-f \in U(R)$ where $f^{2}=f$ and $a f=f a$. By (3), there exists $g^{2}=g \in R$ such that $(a-f)-g \in J(R)$. Then $\bar{g}=\overline{a-f} \in U(\bar{R})$, so $\bar{g}=\overline{1}$. Thus, $(a-1)-f=(a-f)-$ $1 \in J(R)$ with $(a-1) f=f(a-1)$. So $f=e$ by (3).

$(2) \Rightarrow(1)$. Suppose that (2) holds. Then $R$ is clearly an exchange ring. Assume $a-e_{i} \in\left(a^{2}-a\right) R$ where $e_{i}$ is an idempotent for $i=1,2$. Then $a e_{i}=e_{i} a$ by hypothesis. Thus it follows from Lemma 3 that $a-\left(1-e_{i}\right) \in U(R)$ for $i=1,2$, so $1-e_{1}=1-e_{2}$ by (2). That is $e_{1}=e_{2}$. Hence $R$ is a right uniquely exchange ring.

$(1) \Rightarrow(4)$. This is clear.

(4) $\Rightarrow(1)$. Suppose that $a-e_{i} \in\left(a^{2}-a\right) R$ where $e_{i}^{2}=e_{i}$ for $i=1,2$. By Lemma $16, u_{i}:=a-\left(1-e_{i}\right) \in U(R)$, and so $\overline{1}=\bar{u}_{i}=\bar{a}-\left(\overline{1}-\bar{e}_{i}\right)$ for $i=1$, 2 . Hence $\bar{e}_{1}=\bar{e}_{2}$, that is $e_{1}-e_{2} \in J(R)$. By (4), $e_{1} e_{2}=e_{2} e_{1}$. So $e_{1}\left(1-e_{2}\right)=\left(e_{1}-e_{2}\right)\left(1-e_{2}\right) \in J(R)$ is an idempotent. It follows that $e_{1}=e_{1} e_{2}$. Similarly, $\left(1-e_{1}\right) e_{2}=\left(1-e_{1}\right)\left(e_{1}-e_{2}\right) \in J(R)$ is an idempotent, so $e_{2}=e_{1} e_{2}$. Hence $e_{1}=e_{2}$. 
It is worth noting that, in a right uniquely exchange ring $R, a-e \in\left(a^{2}-a\right) R$ with $e^{2}=e$ implies $a-e \in R\left(a^{2}-a\right)$. Thus, the next result is an immediate consequence of Theorem 17.

COROLLARY 18. The following are equivalent for a ring $R$ :

(1) $R$ is a left and right uniquely exchange ring.

(2) $R$ is semi-boolean and, for each $a \in R$, the idempotents $e$ satisfying $a-e \in$ $\left(a^{2}-a\right) R \cup R\left(a^{2}-a\right)$ commute with each other.

The next example shows that a semi-boolean ring need not be a right uniquely exchange ring.

EXAMPLE 19. Let $R=\mathbb{T}_{2}\left(\mathbb{Z}_{4}\right)$. Then $R$ is semi-boolean, but it is neither a right nor a left uniquely exchange ring.

Proof. It is clear that $R$ is a semi-boolean ring. It follows by Theorem 21 that $R$ is neither a right nor a left uniquely exchange ring.

Later we will see that right uniquely exchange rings need not be left uniquely exchange rings.

5. Extensions of rings. An easy argument proves the following result.

EXAMPLE 20. A direct product $\prod_{i} R_{i}$ of rings is a right uniquely exchange ring if and only if each $R_{i}$ is a right uniquely exchange ring.

The next theorem answers when a triangular matrix ring is a right uniquely exchange ring. Maybe it is worth noting that the ring $S:=\mathbb{T}_{2}(R)$ in part (2) of next theorem has the property that, for each $a \in S, e=a^{2}$ is the only idempotent of $S$ such that $a-e \in\left(a^{2}-a\right) S$.

THEOREM 21. Let $n \geq 2$. The following hold for a ring $R$ :

(1) $\mathbb{T}_{n}(R)$ is not a right (left) uniquely exchange ring for each $n \geq 3$.

(2) $\mathbb{T}_{2}(R)$ is a right (left) uniquely exchange ring iff $R$ is a boolean ring.

Proof. (1) Because of Proposition 11, it suffices to show that $S:=\mathbb{T}_{3}(R)$ is not a right uniquely exchange ring. Let

$$
a=\left(\begin{array}{lll}
0 & 1 & 0 \\
0 & 0 & 1 \\
0 & 0 & 1
\end{array}\right) \quad \text { and } e=\left(\begin{array}{lll}
0 & 0 & 0 \\
0 & 0 & 1 \\
0 & 0 & 1
\end{array}\right)
$$

Then $e^{2}=e$ and

$a-e=\left(\begin{array}{lll}0 & 1 & 0 \\ 0 & 0 & 0 \\ 0 & 0 & 0\end{array}\right)=\left(\begin{array}{ccc}0 & 1 & -1 \\ 0 & 0 & 0 \\ 0 & 0 & 0\end{array}\right)\left(\begin{array}{lll}0 & 0 & 0 \\ 0 & 1 & 0 \\ 0 & 0 & 0\end{array}\right)=\left(a-a^{2}\right)\left(\begin{array}{ccc}0 & 0 & 0 \\ 0 & 1 & 0 \\ 0 & 0 & 0\end{array}\right) \in\left(a^{2}-a\right) S$.

But

$$
a e=\left(\begin{array}{lll}
0 & 0 & 1 \\
0 & 0 & 1 \\
0 & 0 & 1
\end{array}\right) \quad \text { and } e a=\left(\begin{array}{lll}
0 & 0 & 0 \\
0 & 0 & 1 \\
0 & 0 & 1
\end{array}\right)
$$

So $a e \neq e a$. Thus, $S$ is not a right uniquely exchange ring by Theorem 17 . 
(2) ' $\Rightarrow$ '. Suppose that $\mathbb{T}_{2}(R)$ is a right uniquely exchange ring. Because of Proposition 11, it follows that $R$ is a right uniquely exchange ring. Thus, by Theorem $17, R / J(R)$ is boolean. So to show that $R$ is boolean, it suffices to show that $J(R)=0$. Let $r \in J(R)$. Write $a=\left(\begin{array}{ll}r & r \\ 0 & 1\end{array}\right)$ and $e=\left(\begin{array}{ll}0 & 0 \\ 0 & 1\end{array}\right)$. Then $e^{2}=e$ and $a-e=\left(\begin{array}{ll}r & r \\ 0 & 0\end{array}\right)=$ $\left(\begin{array}{cc}r-r^{2} & -r^{2} \\ 0 & 0\end{array}\right)\left(\begin{array}{cc}(1-r)^{-1} & (1-r)^{-1} \\ 0 & 0\end{array}\right) \in\left(a^{2}-a\right) \mathbb{\mathbb { T }}_{2}(R)$. Moreover, $a e=\left(\begin{array}{ll}0 & r \\ 0 & 1\end{array}\right)$ and $e a=\left(\begin{array}{ll}0 & 0 \\ 0 & 1\end{array}\right)$. But, by Theorem 17 , ae $=e a$. So $r=0$.

(2) ' $\Leftarrow$ '. Suppose that $R$ is boolean. Then $S:=\mathbb{T}_{2}(R)$ is an exchange ring. Now assume that $a-e \in\left(a^{2}-a\right) S$ with $e^{2}=e$. We prove that $e$ is uniquely determined by $a$. Further assume that $a-e=\left(a-a^{2}\right) b$ with $b \in S$. Write

$$
a=\left(\begin{array}{cc}
r_{1} & r_{2} \\
0 & r_{3}
\end{array}\right), \quad b=\left(\begin{array}{cc}
s_{1} & s_{2} \\
0 & s_{3}
\end{array}\right), \quad \text { and } \quad e=\left(\begin{array}{cc}
e_{1} & e_{2} \\
0 & e_{3}
\end{array}\right) .
$$

It follows from $a-e=\left(a-a^{2}\right) b$ that $e_{1}=r_{1}, e_{3}=r_{3}$ and $e_{2}=r_{2}+r_{2}\left(1-\left(r_{1}+r_{3}\right)\right) s_{3}$. Thus, since $e^{2}=e$, one obtains

$$
\begin{aligned}
e_{2} & =\left(e_{1}+e_{3}\right) e_{2} \\
& =\left(r_{1}+r_{3}\right)\left[r_{2}+r_{2}\left(1-\left(r_{1}+r_{3}\right)\right) s_{3}\right] \\
& =\left(r_{1}+r_{3}\right) r_{2}+r_{2}\left(r_{1}+r_{3}\right)\left(1-\left(r_{1}+r_{3}\right)\right) s_{3} \\
& =\left(r_{1}+r_{3}\right) r_{2} .
\end{aligned}
$$

So $e=a^{2}$ is uniquely determined by $a$.

The left versions of (1) and (2) can be similarly proved.

The factor rings of a right uniquely exchange ring are right uniquely exchange rings. This follows from the result of Pedersen and Perera [20]. We refer to Ara [2] for the discussion of exchange rings without unity.

LeMma 22 [20, Lemma 1.3]. Let $\pi: R \rightarrow S$ be a surjective morphism between (not necessarily unital) exchange rings, and let $\bar{x}, \bar{y}$ and $\bar{z}$ be elements in $S$ such that with $\bar{e}=\bar{x} \bar{y}$ we have

$$
\bar{y}=\bar{y} \bar{e}, \quad 1-\bar{e}=(1-\bar{x})(1-\bar{z}), \quad(1-\bar{z}) \bar{e}=0 .
$$

For each choice of $x$ in $R$ with $\pi(x)=\bar{x}$ there are then elements $y$ and $z$ in $R$ with $\pi(y)=\bar{y}$ and $\pi(z)=\bar{z}$, such that with $e=x y$ we have

$$
y=y e, \quad 1-e=(1-x)(1-z), \quad(1-z) e=0 .
$$

THEOREM 23. Let $R$ be a right uniquely exchange ring and let $I$ be an ideal of $R$. Then $R / I$ is a right uniquely exchange ring.

Proof. Write $\bar{R}=R / I$ and write $\bar{r}=r+I$ for all $r \in R$. It is well known that the factor ring of an exchange ring is again an exchange ring. So $\bar{R}$ is an exchange ring. Now suppose that for some $\bar{x} \in \bar{R}$ and some $\bar{e}_{i}^{2}=\bar{e}_{i} \in \bar{R}(i=1,2)$, there are $\bar{y}_{i}, \bar{z}_{i} \in \bar{R}$ $(i=1,2)$ such that

$$
\bar{e}_{i}=\bar{x} \bar{y}_{i} \text { with } \bar{y}_{i}=\bar{y} \bar{e}_{i} \text {, and } 1-\bar{e}_{i}=(1-\bar{x})\left(1-\bar{z}_{i}\right) \text { with }\left(1-\bar{z}_{i}\right) \bar{e}_{i}=0 \text {. }
$$

Then, by Lemma 22 , there exist $y_{i}^{\prime}, z_{i}^{\prime} \in R(i=1,2)$ with ${\overline{y^{\prime}}}_{i}=\bar{y}_{i}$ and ${\overline{z^{\prime}}}_{i}=\bar{z}_{i}$, such that with $f_{i}=x y_{i}^{\prime}$ we have $y_{i}^{\prime}=y_{i}^{\prime} f_{i}, \quad 1-f_{i}=(1-x)\left(1-z_{i}^{\prime}\right), \quad\left(1-z_{i}^{\prime}\right) f_{i}=0$. Thus, $f_{i}^{2}=f_{i}$. 
Because $R$ is a right uniquely exchange ring, it must be that $f_{1}=f_{2}$. So $\bar{e}_{1}=\bar{f}_{1}=\bar{f}_{2}=$ $\bar{e}_{2}$. Hence $\bar{R}$ is a right uniquely exchange ring.

THEOREM 24. The following are equivalent for a ring $R$ :

(1) $R[[x]]$ is a right uniquely exchange ring.

(2) $R[x] /\left(x^{n}\right)$ is a right uniquely exchange ring for all $n \geq 1$.

(3) $R[x] /\left(x^{n}\right)$ is a right uniquely exchange ring for some $n \geq 2$.

(4) $R[x] /\left(x^{2}\right)$ is a right uniquely exchange ring.

(5) $R$ is an abelian, right uniquely exchange ring.

(6) $R$ is a uniquely clean ring.

In this case, $R[[x]]$ is a uniquely clean ring.

Proof. $\quad(1) \Rightarrow(2)$. For any $n \geq 1, \quad R[x] /\left(x^{n}\right) \cong R[[x]] /\left(x^{n}\right)$, so (2) holds by Theorem 23.

(2) $\Rightarrow$ (3). This is obvious.

(3) $\Rightarrow$ (4). If $n \geq 2$, then $R[x] /\left(x^{2}\right)$ is a homomorphic image of $R[x] /\left(x^{n}\right)$. So the implication follows by Theorem 23 .

(4) $\Rightarrow(5)$. Let $S=R[x] /\left(x^{2}\right)$. Then $S=\{r+s x: r, s \in R\}$ with $x^{2}=0$. Since $R$ is a homomorphic image of $S$, it follows from (4) that $R$ is a right uniquely exchange ring by Theorem 23. Now let $r, e \in R$ with $e^{2}=e$, and let $a=e+(1+e r-r e) x \in S$. Then $a-a^{2}=(1-2 e) x$. Consider two idempotents $e$ and $f:=e+($ re - ere $) x$ of $S$. We have

$$
a-e=(1+e r-r e) x=[(1-2 e) x][(1-2 e)(1+e r-r e)+x] \in\left(a-a^{2}\right) S
$$

and

$a-f=(1+e r-2 r e+e r e) x=[(1-2 e) x][(1-2 e)(1+e r-2 r e+e r e)+x] \in\left(a-a^{2}\right) S$.

Because $S$ is a right uniquely exchange ring, one obtains $e=f$ and $a e=e a$ (by Theorem 17). This gives $r e=e r e$ and $e r e-r e=e r-e r e$. It follows that $e r=r e$.

$(5) \Rightarrow(6)$. This follows by Theorem 17(4).

$(6) \Rightarrow(1)$. Suppose that $R$ is uniquely clean. Then by [16, Corollary 10$], R[[x]]$ is uniquely clean, so it is a right uniquely exchange ring.

Theorem 24 shows that a power series ring is a right uniquely exchange ring if and only if it is uniquely clean. Let $\sigma$ be an endomorphism of $R$. Notice that, by [16, Example 9], $R[[x ; \sigma]]$ is uniquely clean iff $R$ is uniquely clean and $\sigma(e)=e$ for all $e^{2}=e \in R$. The next example gives an interesting contrast to Theorem 24 for skew power series rings.

EXAMPLE 25 . Let $R=\mathbb{Z}_{2} \oplus \mathbb{Z}_{2}$ and let $\sigma: R \rightarrow R,(r, s) \mapsto(s, r)$.

(1) $R[x ; \sigma] /\left(x^{3}\right)$ is a right uniquely exchange ring, and so is $R[x ; \sigma] /\left(x^{2}\right)$.

(2) $R[x ; \sigma] /\left(x^{4}\right)$ is not a right uniquely exchange ring; hence $R[x ; \sigma] /\left(x^{n}\right)(n \geq 4)$ and $R[[x ; \sigma]]$ are not right uniquely exchange rings.

Proof. (1) Let $S=R[x ; \sigma] /\left(x^{3}\right)$. Then $J(S)=x S$, and $S$ is clearly an exchange ring. By Example 7, for any $b \in x S, b$ and $1-b$ are right uniquely exchange elements in $S$; so we only need to show that $(1,0)+b$ and $(0,1)+b$ are right uniquely exchange elements in $S$. Since $(0,1)+b=1-[(1,0)+b]$, it suffices to show that 
$(1,0)+b$ is a right uniquely exchange element in $S$ by Example 7. Write $b=$ $r x+s x^{2}$ and let $a=(1,0)+b=(1,0)+r x+s x^{2}$. Then $a^{2}=(1,0)+r x+r \sigma(r) x^{2}$ and $a^{2}-a=[r \sigma(r)+s] x^{2}$. Suppose that $a-e \in\left(a^{2}-a\right) S$ where $e^{2}=e=e_{0}+e_{1} x+$ $e_{2} x^{2} \in S$. Thus for some $b=b_{0}+b_{1} x+b_{2} x^{2}$,

$$
\begin{aligned}
a-e & =\left(a^{2}-a\right) b=[r \sigma(r)+s] x^{2} \cdot\left(b_{0}+b_{1} x+b_{2} x^{2}\right) \\
& =[r \sigma(r)+s] b_{0} x^{2} .
\end{aligned}
$$

It follows that $e_{0}=(1,0), e_{1}=r$ and $s-e_{2}=[r \sigma(r)+s] b_{0}$. So $e=(1,0)+r x+$ $\left[s+(r \sigma(r)+s) b_{0}\right] x^{2}$. Thus, $e^{2}=(1,0)+r x+r \sigma(r) x^{2}$. Since $e^{2}=e, e=(1,0)+r x+$ $r \sigma(r) x^{2}$ is uniquely determined by $a$. So $a$ is a right uniquely exchange element in $S$. Hence $S$ is a right uniquely exchange ring. As an image of $S, R[x ; \sigma] /\left(x^{2}\right)$ is a right uniquely exchange ring.

(2) Let $S=R[x ; \sigma] /\left(x^{4}\right)$, and let $a=(1,0)+x+(1,0) x^{2}+x^{3}$ and $e=(1,0)+$ $x+x^{2}+(0,1) x^{3}$ be in $S$. By direct calculation, one obtains that $e^{2}=e$ and

$$
\begin{aligned}
a-e & =(0,1) x^{2}+(1,0) x^{3} \\
& =\left[(0,1) x^{2}+x^{3}\right](1+x) \\
& =\left(a^{2}-a\right)(1+x) \in\left(a^{2}-a\right) S .
\end{aligned}
$$

But $e a=(1,0)+x+x^{2}+(0,1) x^{3}$ and $a e=(1,0)+x+x^{2}$. So $a e \neq e a$. Hence $S$ is not a right uniquely exchange ring by Theorem 17 . For any $n \geq 4, S$ is an image of $R[x ; \sigma] /\left(x^{n}\right)$ and the latter is an image of $R[[x ; \sigma]]$. It follows that $R[x ; \sigma] /\left(x^{n}\right)$ and $R[[x ; \sigma]]$ are not right uniquely exchange rings.

QUeSTION 2. Let $\sigma$ be an endomorphism of a ring $R$ and $n \geq 2$ be an integer. When is $R[[x ; \sigma]]$ a right uniquely exchange ring? When is $R[x ; \sigma] /\left(x^{n}\right)$ a right uniquely exchange ring?

6. The asymmetry. The definition of an exchange ring is left-right symmetric. Here we prove that right uniquely exchange rings need not be left uniquely exchange rings.

Let $R, S$ be rings and $M$ be an $(R, S)$-bimodule. Consider the formal triangular $\operatorname{ring} T=\left(\begin{array}{cc}R & M \\ 0 & S\end{array}\right)$.

LEMMA 26. Given $T=\left(\begin{array}{cc}R & M \\ 0 & S\end{array}\right)$, the following hold:

(1) If $T$ is a right uniquely exchange ring, then $R, S$ are right uniquely exchange rings and $J(R) M=0$.

(2) If $T$ is a left uniquely exchange ring, then $R, S$ are left uniquely exchange rings and $M J(S)=0$.

Proof. We prove (1) only. Suppose that $T$ is a right uniquely exchange ring. Then $R, S$ are right uniquely exchange rings by Proposition 11. For $a \in J(R)$ and $m \in M$, let $\alpha=\left(\begin{array}{cc}a & m \\ 0 & 1\end{array}\right)$ and $e=\left(\begin{array}{cc}0 & m \\ 0 & 1\end{array}\right)$. Then $e^{2}=e$ and $\alpha-e=\left(\begin{array}{ll}a & 0 \\ 0 & 0\end{array}\right)=$ $\left(\begin{array}{cc}a(1-a) & -a m \\ 0 & 0\end{array}\right)\left(\begin{array}{cc}(1-a)^{-1} & 0 \\ 0 & 0\end{array}\right)=\left(\alpha-\alpha^{2}\right)\left(\begin{array}{cc}(1-a)^{-1} & 0 \\ 0 & 0\end{array}\right) \in\left(\alpha-\alpha^{2}\right) T$. By Theorem 17, $\alpha e=e \alpha$. It follows that $a m=0$.

THEOREM 27. Given $T=\left(\begin{array}{cc}R & M \\ 0 & S\end{array}\right)$, the following are equivalent:

(1) $T$ is a uniquely exchange ring.

(2) $R$ and $S$ are uniquely exchange rings and $J(R) M=0=M J(S)$. 
Proof. (1) $\Rightarrow$ (2). This is by Lemma 26 .

$(2) \Rightarrow(1)$. We prove $T$ to be a right uniquely exchange ring only. Clearly, $T$ is an exchange ring. Let $\alpha=\left(\begin{array}{cc}a & m \\ 0 & b\end{array}\right) \in T$. Suppose that $\alpha-\eta_{i}=\left(\alpha-\alpha^{2}\right) \beta_{i}$, where $\eta_{i}=$ $\left(\begin{array}{cc}e_{i} & m_{i} \\ 0 & f_{i}\end{array}\right)$ is an idempotent of $T$ and $\beta_{i}=\left(\begin{array}{cc}r_{i} & k_{i} \\ 0 & s_{i}\end{array}\right) \in T$ for $i=1,2$. Thus, one obtains

$$
\begin{aligned}
\left(\begin{array}{cc}
a-e_{i} & m-m_{i} \\
0 & b-f_{i}
\end{array}\right) & =\left(\begin{array}{cc}
a-a^{2} & m-a m-m b \\
0 & b-b^{2}
\end{array}\right)\left(\begin{array}{cc}
r_{i} & k_{i} \\
0 & s_{i}
\end{array}\right) \\
& =\left(\begin{array}{cc}
\left(a-a^{2}\right) r_{i} & \left(a-a^{2}\right) k_{i}+(m-a m-m b) s_{i} \\
0 & \left(b-b^{2}\right) s_{i}
\end{array}\right) \\
& =\left(\begin{array}{cc}
\left(a-a^{2}\right) r_{i} & (m-a m-m b) s_{i} \\
0 & \left(b-b^{2}\right) s_{i}
\end{array}\right),
\end{aligned}
$$

because $\left(a-a^{2}\right) k_{i}=0$ (as $\left.a-a^{2} \in J(R)\right)$. Thus, we have

$$
\begin{aligned}
a-e_{i} & =\left(a-a^{2}\right) r_{i} \\
b-f_{i} & =\left(b-b^{2}\right) s_{i} \\
m-m_{i} & =(m-a m-m b) s_{i} .
\end{aligned}
$$

From $\eta_{i}^{2}=\eta_{i}$, it follows that

$$
e_{i}^{2}=e_{i}, \quad f_{i}^{2}=f_{i} \quad \text { and } \quad m_{i}=e_{i} m_{i}+m_{i} f_{i} .
$$

Thus, since $R$ and $S$ are uniquely exchange rings, we obtain that $e:=e_{1}=e_{2}$ and $f:=f_{1}=f_{2}$. Since $\left(a-a^{2}\right) r_{i} \in J(R)$ and $\left(b-b^{2}\right) s_{i} \in J(S)$, am $=e m$ and $m b=m f$ by (6.1) and our assumption. So $m_{i}=m-(m-a m-m b) s_{i}=m-(m-e m-m f) s_{i}$, and hence $e m_{i}=e m-e(m-e m-m f) s_{i}=e m+e m f s_{i}$ and $m_{i} f=m f-(m-e m-$ $m f) s_{i} f$. Now from (6.2) we have for $i=1,2$ that

$$
\begin{aligned}
m_{i} & =e_{i} m_{i}+m_{i} f_{i}=e m_{i}+m_{i} f \\
& =e m+e m f s_{i}+m f-(m-e m-m f) s_{i} f \\
& =e m+m f+e m\left(f s_{i}-s_{i} f\right)+m(f-1) s_{i} f \\
& =e m+m f,
\end{aligned}
$$

because both $f s_{i}-s_{i} f$ and $(f-1) s_{i} f$ are in $J(S)$. So $m_{1}=m_{2}$ and hence $\eta_{1}=\eta_{2}$.

Our concluding example gives a right uniquely exchange ring that is not a left uniquely exchange ring.

EXAMPLE 28. Let $T=\left(\begin{array}{cc}R & M \\ 0 & S\end{array}\right)$, where $R=\mathbb{Z}_{2}, S=\mathbb{Z}_{2}[[x]]$ and $M=\mathbb{Z}_{2}[[x]]$. Then $T$ is a right uniquely exchange ring that is not a left uniquely exchange ring.

Proof. Since $J(S)=x S$ and $M J(S) \neq 0, T$ is not a left uniquely exchange ring by Lemma 26. $T$ is clearly an exchange ring. To show that $T$ is a right uniquely exchange ring, let $\alpha=\left(\begin{array}{cc}a & f(x) \\ 0 & g(x)\end{array}\right) \in T$; we show that there exists a unique $\eta^{2}=\eta \in T$ such that $\alpha-\eta \in\left(\alpha-\alpha^{2}\right) T$. Because of Example 7, we may assume that $\alpha^{2} \neq \alpha$ and $\alpha \notin J(T)=\left(\begin{array}{cc}0 & M \\ 0 & x S\end{array}\right)$. Thus, there are two cases: (i) $a=0$ and $g(x) \in U(S)$; and (ii) $a=1$. 
Write $\alpha-\eta=\left(\alpha-\alpha^{2}\right)\left(\begin{array}{cc}a_{0} & f_{0}(x) \\ 0 & g_{0}(x)\end{array}\right)$. Then

$$
\begin{aligned}
\eta & =\left(\begin{array}{ll}
a & f(x) \\
0 & g(x)
\end{array}\right)-\left(\begin{array}{cc}
a-a^{2} & f(x)(1-a-g(x)) \\
0 & g(x)(1-g(x))
\end{array}\right)\left(\begin{array}{cc}
a_{0} & f_{0}(x) \\
0 & g_{0}(x)
\end{array}\right) \\
& =\left(\begin{array}{cc}
a-\left(a-a^{2}\right) a_{0} & f(x)\left[1-(1-a-g(x)) g_{0}(x)\right]-\left(a-a^{2}\right) f_{0}(x) \\
0 & g(x)\left[1-(1-g(x)) g_{0}(x)\right]
\end{array}\right) .
\end{aligned}
$$

Case 1. $a=0$ and $g(x) \in U(S)$. Then

$$
\eta=\left(\begin{array}{ll}
0 & f(x)\left[1-(1-g(x)) g_{0}(x)\right] \\
0 & g(x)\left[1-(1-g(x)) g_{0}(x)\right]
\end{array}\right)
$$

and

$$
\eta^{2}=\left(\begin{array}{cc}
0 & f(x) g(x)\left[1-(1-g(x)) g_{0}(x)\right]^{2} \\
0 & g(x)^{2}\left[1-(1-g(x)) g_{0}(x)\right]^{2}
\end{array}\right)
$$

Being both an idempotent and a unit in $S, g(x)\left[1-(1-g(x)) g_{0}(x)\right]$ must be 1 . So $1-(1-g(x)) g_{0}(x)=g(x)^{-1}$ and hence $\eta=\left(\begin{array}{cc}0 & f(x) g(x)^{-1} \\ 0 & 1\end{array}\right)$.

Case 2. $a=1$. Then

$$
\eta=\left(\begin{array}{cc}
1 & f(x)\left[1+g(x) g_{0}(x)\right] \\
0 & g(x)\left[1-(1-g(x)) g_{0}(x)\right]
\end{array}\right)
$$

and

$$
\eta^{2}=\left(\begin{array}{cc}
1 & f(x)\left[1+g(x) g_{0}(x)\right]\left[1+g(x)\left(1-(1-g(x)) g_{0}(x)\right)\right] \\
0 & g(x)^{2}\left[1-(1-g(x)) g_{0}(x)\right]^{2}
\end{array}\right) .
$$

If $g(x) \in U(S)$, then $g(x)\left[1-(1-g(x)) g_{0}(x)\right]=1$ as above, and this gives $f(x)[1+$ $\left.g(x) g_{0}(x)\right]=0$. So $\eta=\left(\begin{array}{cc}1 & 0 \\ 0 & 1\end{array}\right)$.

If $g(x) \notin U(S)$, then $g(x) \in J(S)$. Thus, $g(x)\left[1-(1-g(x)) g_{0}(x)\right]$ is both an idempotent and an element in $J(S)$, so it must be that $g(x)\left[1-(1-g(x)) g_{0}(x)\right]=0$. This also gives that $g(x)=g(x)(1-g(x)) g_{0}(x)$. It follows that $g(x) g_{0}(x)=g(x)(1-$ $g(x))^{-1}$. So $\eta=\left(\begin{array}{ll}1 & f(x)\left[1+g(x)(1-g(x))^{-1}\right] \\ 0 & 0\end{array}\right)$. The proof is now complete.

Acknowledgements. The authors are grateful to the referee for his valuable comments. In particular, Example 15 was suggested to the authors by the referee. Part of the work was carried out when the second author was visiting the National Taiwan University sponsored by NCTS of Taipei. He gratefully acknowledges the financial support from NCTS and kind hospitality from the host university. The research of the first author was supported by NSC of Taiwan, and that of the second author by NSERC of Canada.

\section{REFERENCES}

1. D. D. Anderson and V. P. Camillo, Commutative rings whose elements are a sum of a unit and idempotent, Comm. Algebra 30(7) (2002), 3327-3336.

2. P. Ara, Extensions of exchange rings, J. Algebra 197(2) (1997), 409-423. 
3. P. Ara, Stability properties of exchange rings, International Symposium on Ring Theory, (Kyongju, 1999), 23-42, Trends Math., Birkhaüser Boston, Boston, MA, 2001.

4. P. Ara, K. R. Goodearl, K. C. O'Meara and E. Pardo, Separative cancellation for projective modules over exchange rings, Israel J. Math. 105 (1998), 105-137.

5. V. P. Camillo and H.-P. Yu, Exchange rings, units and idempotents, Comm. Algebra 22 (1994), 4737-4749.

6. J. Chen, W. K. Nicholson and Y. Zhou, Group rings in which every element is uniquely the sum of a unit and an idempotent, J. Algebra 306(2) (2006), 453-460.

7. P. Crawley and B. Jonsson, Refinements for infinite decompositions of algebraic systems, Pacific J. Math. 14 (1964), 797-855.

8. N. Dubrovin, P. Př́ihoda, and G. Puninski, Projective modules over the GerasimovSakhaev counter example, J. Algebra 319(8) (2008), 3259-3279.

9. M. Harada, Factor categories with applications to direct sum decomposition of modules, Lecture Notes in Pure and Applied Math. 88, Marcel Dekker, New York, 1983.

10. T. Y. Lam, A crash course on stable range, cancellation, substitution and exchange, J. Algebra Appl. 3 (2004), 301-343.

11. J. Levitzki, On the structure of algebraic algebras and related rings, Trans. AMS. 74 (1953), 384-409.

12. S. H. Mohamed and B. J. Müller, Continuous and discrete modules, London Math. Soc. Lectures Note Series 147, Cambridge University Press, Cambridge, UK, 1990.

13. W. K. Nicholson, Lifting idempotents and exchange rings, Trans. AMS. 229 (1977), $269-278$

14. W. K. Nicholson, On exchange rings, Comm. Algebra 25(6) (1997), 1917-1918.

15. W. K. Nicholson, Strongly clean rings and Fitting's lemma, Comm. Algebra 27 (1999), $3583-3592$.

16. W. K. Nicholson and Y. Zhou, Rings in which elements are uniquely the sum of an idempotent and a unit, Glasgow Math. J. 46 (2004), 227-236.

17. W. K. Nicholson and Y. Zhou, Strong lifting, J. Algebra 285(2) (2005), 795-818.

18. W. K. Nicholson and Y. Zhou, Clean general rings, J. Algebra 291(1) (2005), 297-311.

19. K. Oshiro and S. T. Rizvi, The exchange property of quasi-continuous modules with the finite exchange property, Osaka J. Math. 33 (1996), 217-234.

20. G. K. Pedersen and P. Perera, Inverse limits of rings and multiplier rings, Math. Proc. Camb. Phil. Soc. 139 (2005), 207-228.

21. E. Sánchez Campos, On strongly clean rings, 2002 (unpublished).

22. R. B. Warfield Jr., Exchange rings and decompositions of modules, Math. Ann. 199 (1972), 31-36.

23. B. Zimmermann-Huisgen and W. Zimmermann, Classes of modules with the exchange property, J. Algebra 88 (1984), 416-434. 